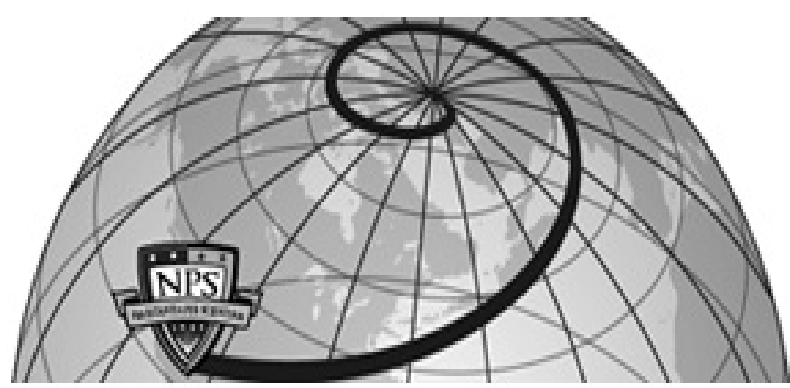

Calhoun: The NPS Institutional Archive DSpace Repository

\title{
Testing Adaptive Probabilistic Software Components in Cyber Systems
}

\section{Luqi; Jacoby, Grant}

Testing Adaptive Probabilistic Software Components in Cyber Systems, with G. Jacoby, Springer LNCS 6662, pp. 228-238, ISBN 978-3-642-21291-8.

https://hdl.handle.net/10945/42347

This publication is a work of the U.S. Government as defined in Title 17, United States Code, Section 101. Copyright protection is not available for this work in the United States.

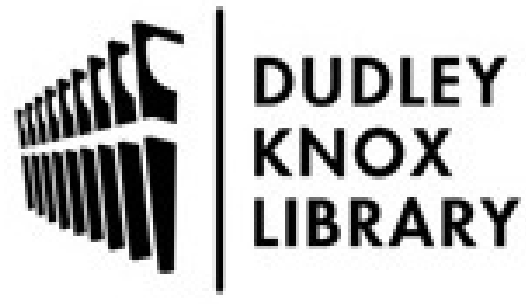

http://www.nps.edu/library
Calhoun is the Naval Postgraduate School's public access digital repository for research materials and institutional publications created by the NPS community. Calhoun is named for Professor of Mathematics Guy K. Calhoun, NPS's first appointed -- and published -- scholarly author.

Dudley Knox Library / Naval Postgraduate School 411 Dyer Road / 1 University Circle Monterey, California USA 93943 


\title{
Testing Adaptive Probabilistic Software Components in Cyber Systems
}

\author{
Luqi $^{1}$ and Grant Jacoby ${ }^{2}$ \\ ${ }^{1}$ Naval Postgraduate School \\ ${ }^{2}$ United States Military Academy
}

\section{Introduction}

This paper addresses improved principles for verification and validation to establish confidence in robustness of adaptive software systems, to include uncertainty with respect to cyber environment and dynamics of internal system configuration. It applies to component based systems with probabilistic decision making at multiple levels and bridges the gap between checking the correctness of a single component and validating systems composed of many components.

Robust adaptive software design requires substantial architectural support. Sound architectural models with adaptive probabilistic software components in cyber systems and associated quality assurance methods collectively gain the ability to replace bits of systems while maintaining system dependability [1]. We focus on system design with recorded rationale to make the testing of adaptive probabilistic software components in cyber systems possible.

This requires a shift from scenario-based testing to architecture-based quality assurance [2, 3, 4], along with a shift from code-based adaptation to architecturebased adaptation [5, 6, 7]. A good cyber system architecture would have associated dependability properties that express stable system requirements, requirements on the subsystems, and a sound software evolution model capturing the design rationale [8]. For systems that are supposed to be automatically adaptive, the adaptation is accomplished by structures explicitly visible in the software architecture. The architecture itself would provide some degree of dependability guarantees, regardless of specific configuration. Testing and analysis would be applied to a suitably specified architectural model in addition to the system implementation, as described in section 4 . Realizing this vision will require a more precise and detailed description of the architecture than is commonly developed in current practice

Robust composite systems can be composed of components in a disciplined manner, according to a carefully designed software architecture. A software architecture consists of:

1. A set of components

2. An interconnection pattern for the components, and

3. A set of constraints on the components and connections.

The constraints typically express various kinds of requirements associated with the entire systems as well as with the components and connections of the architecture $[9,22]$. 
An architecture model is an explicit and precise definition of an architecture that specifies the details of its structure and the constraints. Architecture models play a crucial role in the design and testing of flexible systems, especially for those that are supposed to be self-adapting at runtime. In this context, the constraints associated with the entire system express invariant requirements that are supposed to be met in all the configurations that can be reached via adaptation transitions. The constraints on the components and the connections express the common principles of operation shared by all of those configurations.

In a well-designed adaptive system the invariant requirements can be established based solely on the constraints associated with the components and the connections. This enables the system to operate properly regardless of which concrete components are chosen to fill each component slot in the architecture model, as long as each concrete component satisfies the constraints associated with its component slot. In this vision, each component slot has a set of compatible components, all of which satisfy the role requirements associated with a given slot in the architecture, but the individual components may differ in the specifics of their behavior within the envelope defined by the role requirements. This set may be further organized according to feature parameters that characterize the degree of component behavior variability that can be accommodated by the architecture model.

The individual components associated with a given slot in the architecture have to be certified with respect to the role requirements associated with the slot. All variants of these components must satisfy the minimum common role requirements. In cases where alternative slot-compatible components have different specialized capabilities, the role requirements may include corresponding specialized requirement clauses that are conditioned on the particular feature parameter values that call out the subrequirements for those specialized capabilities.

\section{How Testing for Adaptive Systems Differs from Traditional Testing}

Traditional software testing techniques like scenario-based integration testing are commonly used for assessing dependability of today's software systems. These techniques are strongly dependent on a particular system configuration and its platform. A major drawback is that when the system configuration or its platform changes, it is necessary to reconstruct the test cases and rerun them. Plugging in a new software component will lead to a completely different system and will likely invalidate previous test results, while changes to the cyber environment may reduce the effective coverage of the test scenarios previously used. Therefore, these techniques are not effective for testing adaptive probabilistic software components in cyber systems where dynamic system configuration and frequent changes are the norm.

Cyber-physical systems are an important special case of adaptive systems [10]. Cyber-Physical Systems (CPS) are integrations of computation and physical processes. Embedded computers and networks monitor and control the physical processes, with feedback loops where physical processes affect computations and vice versa. The technology builds on the older (but still very young) discipline of embedded systems, computers and software embedded in devices whose principle mission is not 
computation, such as cars, toys, medical devices, and scientific instruments. CPS integrate the dynamics of the physical processes with those of the software and networking, providing abstractions and modeling, design, and analysis techniques for the integrated whole. [28].

A cyber-physical system consists of software, computers, and physical components. The software in such systems is typically used to control the physical parts in a way to achieve given goals such as safety concerns, maximizing system utility, and minimizing costs. This control is typically based on sensor readings, and signals that control actuators, both of which have finite accuracy and delay. In large scale contexts, there are generally a variety of goals to be achieved, a variety of physical components to control and coordinate, multiple available sensors and actuators, environmental conditions cannot be perfectly predicted, and all physical components are subject to failure. In many contexts of interest, the cyber-physical systems are expected to operate in inhospitable and possibly hostile environments $[8,11]$.

A similar problem also occurs when the application has to be used in a cyber environment different from the one for which it was originally designed and tested, which is expected to be common for reusable adaptive probabilistic systems and components. This raises an important concern since flexible systems are subjected to frequent changes. In the context of automatically adaptive systems, the situation is even more severe, since adaptation is supposed to happen at runtime, rapidly, and with little or no human intervention. This does not provide enough time for traditional test and evaluation procedures, which are typically time consuming, labor intensive, and dependent on human expertise. We therefore seek techniques that can be applied in advance without detailed knowledge of the specifics of the new configuration and the new operating environment. We need to find principles and methods for quality assurance that can apply to a family of possible new configurations and a family of possible cyber environments simultaneously [12].

Traditional quality assurance approaches rely heavily on testing methods that assume system environment and system configuration are fixed and known prior to testing. These assumptions are invalid for adaptive complex probabilistic systems. Noisy data, deception, and surprise attack tactics are likely for many critical and military systems, making system environment uncertain.

System adaptation and machine learning are inconsistent with keeping system configuration fixed. We propose to model these phenomena as the choice between different configurations that share the same principles of operation and architecture, but differ in specific components that can be compatibly plugged into corresponding slots in the architecture. Probabilistic systems can be also be modeled in this framework, as nondeterministically choosing between components at runtime, where each of the alternative components realizes a different decision strategy. An adaptive system must be designed so that it can continue to operate properly despite replacement of components with other plug-compatible components that may differ in the details of their behavior and capabilities. For example, this strategy is applicable when a variety of robotic platforms are built from a designated set of plug-compatible electronic components, each of which has its own specialized purpose and has been designed to accomplish a specific aspect of a complex mission [29]. 
Fully realizing the dependable architecture vision thus requires new paradigms for both system synthesis and quality assurance. We propose such a paradigm here, based on the concepts of mathematical dependability contracts, interchangeable software parts, and computer-aided enforcement of dependability contracts, both during $\mathrm{V} \& \mathrm{~V}$ and at runtime. For adaptive systems, a dependable architecture includes an explicit adaptation model, with its own requirements, structure, principles of operation, and desired dependability properties. For probabilistic systems, requirements will be expressed in terms of constraints that admit multiple, non-deterministic outcomes. Deterministic systems become a special case, in which the constraints are tight enough to admit only a single behavior in each situation. We assume that multicomponent systems will be designed using adaptive architectures that define the roles of the individual components along with the protocols for the interactions among the components and parameterized goals that are to be achieved by the interactions, using a refinement of the framework described in [13].

We focus primarily on the V \& V aspect, but include aspects of synthesis in cases where constrained design techniques that guarantee certain aspects of correctness by construction via special-purpose design rules, analysis and checking at design time are more effective than methods that seek to certify arbitrary unconstrained designs.

Current approaches to system development and testing are more analogous to individual craftsmanship than they are to modern concepts of mass production and interchangeable parts. Craftsmen used to build things by individually tuning mating parts until they properly fit together. In such a context, designs could be relatively informal and relatively rough. Current software testing practices work this way, particularly for integration testing. Such approaches are not feasible for adaptive systems because the number of possible configurations is much too large (exponential in the number of independently adaptable components) to feasibly test them all in advance.

In modern mass production environments, parts are built to standards with precisely specified tolerances, and it is up to the designer to determine and verify the tolerances necessary to make the design work for any combination of parts that meet the specified tolerances. For example, modern audio systems are designed this way. There exist specific standards for audio specifying how things need to fit together in order for components from different vendors to work together effectively, and components are tested based on these standards, without knowledge of the specifics of the other components they will be connected to. Determining the tolerances necessary to make this work is difficult in general, and current practice depends on highly skilled individuals to get the standards right. However, standards for audio system components can be relatively simple and manageable via relatively informal processes only because the requirements for stereo systems are very simple and insensitive to the meaning of the signals they are processing. An audio system is not concerned about whether it is playing a song or the news. This simplification does not apply to most software systems.

\section{The Scientific Problem}

We are seeking analogous synthesis and quality assurance techniques for systems involving software. For software systems, whose behavior is usually sensitive to the 
meaning of the data, new types of standards will be needed to accomplish a similar function, along with scientific methods for designing and checking the standards to ensure the system will meet its dependability properties, as well as checking conformance of individual components to the standards. This additional degree of rigor is needed for software standards intended to meet specific requirements despite varied choices of components (system adaptation) or nondeterministic variations in the behavior of a single component (probabilistic systems) because such standards are considerably more complex than the standards supporting mass production for electronic or mechanical systems. We are studying principles, models, and methods to enable general static analysis techniques to be combined with testing methods that can support statistically significant conclusions about conformance of components to architecture standards. Targeted static analyses range from techniques involving general mathematical proofs requiring human assistance to special purpose algorithmic methods that can be completely automated for complex applications.

One context in which the proposed approach can be employed is in the design of interchangeable components for robotic platforms. Here the focus is on cooperative robotic platforms where payloads, sensors and tasks are divided into various specialized modular platforms. The modular specialized platforms can then be assembled as a team, custom tailored for the various mission requirements. Decomposing the complex task sequences required of autonomous robots into a hierarchy of increasingly sophisticated control systems provides a powerful method for escalating a robot's degree of autonomy [29]. Analogous synthesis and quality assurance techniques in designing the software for this, or different levels of autonomy at different levels of control, is needed to form a hierarchical command control structure that can deploy and orchestrate a network of autonomous robots.

\section{How to Test Adaptive Systems}

The proposed approach to quality assurance for adaptive systems encompasses:

(1) analysis of software architecture with respect to requirements on many possible configurations,

(2) testing software components against required architecture properties driven by both validated statistical models of expected cyber environments and transformed models, and

(3) a combination of testing and analytical methods for verifying non-interference between components.

The transformed models compensate for uncertainty about possible cyber environments by combining alternative possible models and amplifying the frequency of rare events.

A possible quality assurance approach for adaptive systems is illustrated below $[14,15,31]$. 


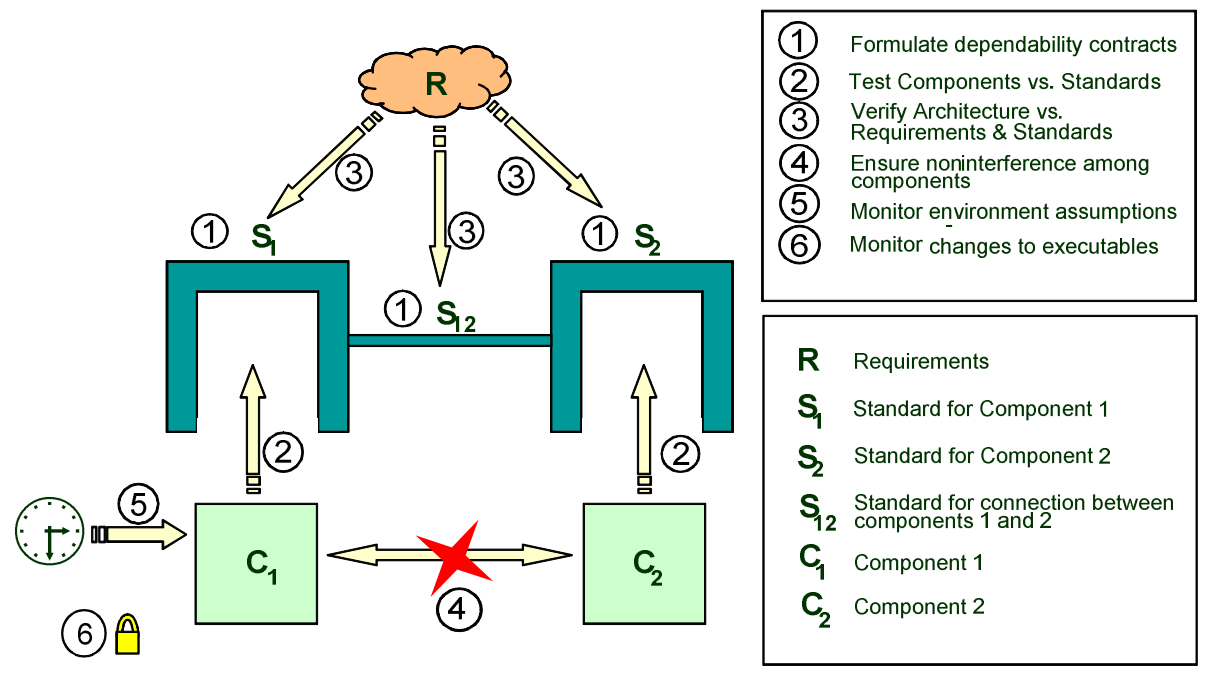

The fundamental operation of such an approach can be outlined as follows:

1.a. System-wide capabilities are characterized by a set of dependability properties that must hold in all acceptable system configurations. These properties comprise the dependability contract for the system as a whole. They become part of the architecture for the system, and serve as the basis for system quality assurance. Dependability contracts are primarily technical rather than legal documents, and they are intended to be checkable via software.

1.b. The designers of the architecture determine the common structure of the system and develop the component-level dependability contracts for the subsystems and connectors. The common structure consists of connection patterns and subsystem slots to which all configurations must conform.

2. The quality assurance team tests each component (subsystem and connector) against its dependability contract. This is envisioned to be an automated process to enable sufficient large sets of test cases for statistically significant conclusions about desirable dependability levels. The cost for this step is proportional to the number of components, and the process must be done once for each version of each atomic component. Technologies for doing this are known, and some of them are starting to be used in practice [30].

3. The quality assurance team checks the structure of the architecture and the dependability contracts for subsystems and connectors to make sure they are strong enough to guarantee the system-wide dependability properties in all possible configurations. This is a new process that uses symbolic analysis techniques. Assuring the feasibility of this step is one of our objectives.

4. The quality assurance team checks components for non-interference. This process is computer-aided. Many of the technologies for this are known and some of them are commonly used, such as data representation hiding enforced by programming language scope rules and type checking. Additional research is needed to get a complete set. This part of the process ensures that 
components that work correctly in isolation will continue to do so when they are connected. Some examples of mechanisms that can cause this type of interference include mutual exclusion constraints, deadlocks, resource constraints (memory and bandwidth limits, etc), timing constraints, out of bounds memory references, etc.

5. The assumptions about the operating environment that the architecture depends on are checked by runtime monitoring. This can be done using BIT (Built-In-Test) technology that is currently in use in some DoD systems. This is recommended for all reusable components.

6. Runtime checks that the machine code actually running in the system corresponds to the source code that was subjected to quality assurance processes, and processes for restoring it to the proper state before execution if these checks fail. These processes are necessary because of the following plausible failure modes:

a. Memory-corrupting bugs-these include out of bounds write operations on arrays and through invalid pointers. Such bugs can cause seemingly innocuous statements to overwrite parts of the program itself at runtime, with unpredictable and potentially catastrophic results.

b. Deliberate cyber-attacks - compromise of system security via network or unauthorized insider access to systems can deliberately modify machine code at run-time.

c. Memory state corruption due to hard radiation, which is plausible in some WMD scenarios, spacecraft, nuclear power systems, scientific and medical applications, etc.

Some approaches to this problem can be found in [15].

Probabilistic methods for testing flexible modular systems to high levels of statistical confidence should be viable for testing complex probabilistic systems. This strategy focuses on using high-fidelity profile-based environment models to automatically generate test cases by sampling from probability density functions or other kinds of probabilistic models that characterize environment parameters. When combined with automated execution and output checking techniques, such models are capable of driving automated software testing, enabling affordable sample sizes large enough to support statistically significant conclusions about system behavior.

\section{Challenges in Validating Statistical Models for a Wide Range of Operating Environments}

Known methods for creating validated statistical models for operating environments include least-squares matching of known parametric distributions, use of the Bayesian Information Criterion or Akaike Information Criterion to choose the best of several models, and non-parametric methods such as kernel density estimation. All of these methods depend on the availability of historical data characterizing a given cyber environment, and assume that the intended cyber environment is known in advance. 
These assumptions are problematic for adaptive systems, because the actual system configuration is not necessarily known in advance. In the context of unprecedented systems, the availability of historical data is also problematic.

Prior work has explored some approaches to modeling of unknown operating environments in the context of software filters for maritime tracks [15], and we are studying, refining and extending these approaches. The legacy operating environment for the previously studied software consisted of large, slow moving ships. The new environment included threats from small fast boats, which are likely to be operated by dynamic non-state organizations such as terrorist groups or smugglers. The specific types of boats involved in such cases are largely unpredictable, as are the likely navigational tactics. The solution approach in this situation was to characterize the operating characteristics of a variety of commercially available small boats, in terms of parameters such as maximum speed, maximum acceleration, maximum deceleration (separately specified because speeding up and slowing down have different mechanisms that produce asymmetric results), and maximum turning rate. These parameters defined the limits of a neighborhood of environmental conditions of reasonable concern. Kernel density estimation techniques were then used to form a composite of the different possibilities for operating conditions. Although the resulting distribution covered a wider range of conditions than each individual possible type of boat was capable of exhibiting, it provided a reasonable characterization of all conditions likely to be of concern, with tails that provided coverage of unexpected but possible conditions. Our initial study was conducted in a relatively simple two-dimensional environment. However, we see no fundamental reason why similar techniques could not be applied in more complex data domains and higher dimensional spaces.

Adaptive systems must deal with equivocality in addition to uncertainty. Here "uncertainty" refers to individually unpredictable events that follow a known probability distribution, and "equivocality" refers to situations where the probability distribution itself is uncertain, or possibly time varying according to unknown patterns. Uncertainty corresponds to the case where the expected operating environment can be characterized in terms of stable types of activities that have a known past history, while equivocality corresponds to more dynamic situations in which unexpected and possibly unprecedented new kinds of events can arise. We have modeled equivocality in the context of possible operating environments under the assumption that "small" perturbations to operating environments are more likely than "large" ones. This leads to systematic exploration of sequences of possible environments produced by perturbing transformations systematically arranged in order of increasing severity. One way to realize this in a way that captures likelihood of occurrence is to repeatedly compose the perturbation transformation to form more severe perturbations. This overall approach is compatible with a strategy that searches the most likely neighborhoods of the expected operating environment first and most thoroughly.

\section{Conclusion}

The usefulness of sound and systematic approaches for achieving dependable, flexible, and cost-effective software has been a focus of scientific interest for many years 
[16-35]. As we move to automatically adaptive software, these types of approaches are appearing to become a necessity rather than a luxury, because manual artisanship simply cannot reach the levels of reliability and speed of adaptation that is called for.

\section{References}

1. Hinchey, M., Vassev, E.: Software Verification of Autonomic Systems Developed with ASSL. In: Monterey Workshop on Modeling, Development, and Verification of Adaptive Systems, Microsoft Research, March 31-April 2, pp. 7-15 (2010)

2. Luqi, Zhang, L., Berzins, V., Qiao, Y.: Documentation Driven Development for Complex Real-Time Systems. IEEE Transactions on Software Engineering 30(12), 936-952 (2004)

3. Qiao, Y., Luqi: Admission Control for Dynamic Software Reconfiguration in Systems of Embedded Systems. In: Proceedings of the 2004 International Conference on Embedded Systems and Applications (ESA 2004), Las Vegas, Nevada, USA, June 21-24, pp. 136142 (2004)

4. Luqi: Transforming Documents to Evolve High-Confidence Systems. In: Proceedings of Workshop on Advances in Computer Science and Engineering, Berkeley, CA, May 6, pp. 71-72 (2006)

5. Oreizy, P., Gorlick, M., Taylor, R., Heimbigner, D., Johnson, G., Medvidovic, N., Quilici, A., Rosenblum, D., Wolf, A.: An Architecture-Based Approach to Self-Adaptive Software. IEEE Intelligent Systems and their Applications 14(3), 54-62 (1999)

6. Garlan, S.W.C., Huang, A.C., Schmerl, B., Steenkiste, P.: Rainbow: Architecture-Based Self-Adaptation with Reusable Infrastructure. Computer 37(10), 46-54 (2004)

7. Calinescu, R.: Reconfigurable Service-Oriented Architecture for Autonomic Computing. International Journal on Advances in Intelligent Syste 2(1), 38-57 (2009)

8. Rajkumar, R., Lee, I., Sha, L., Stankovic, J.: Cyber-Physical Systems: The Next Computing Revolution. In: Proceedings of the 47th Design Automation Conference, pp. 731-736 (2010)

9. Proceedings of the 4th Monterey Workshop on Specification Based Software Architectures, Monterey, California (1995)

10. Proceedings of the 16th Monterey Workshop on Modeling, Development, and Verification of Adaptive Systems, Microsoft Research, March 31-April 2 (2010)

11. Luqi, Kordon, F.: Modeling, Development, and Verification of Adaptive Systems. In: Proceedings of the Monterey Workshop on Modeling, Development, and Verification of Adaptive Systems, Microsoft Research, March 31-April 2 (2010)

12. Sztipanovits, J.: Software Verification for Adaptive Systems: is it Easier or Harder? In: Proceedings of the Monterey Workshop on Modeling, Development, and Verification of Adaptive Systems, Microsoft Research, March 31-April 2 (2010)

13. Luqi: Dependable Software Architecture Based on Quantifiable Compositional Model, AFOSR Final Report (November 2007)

14. Berzins, V., Rodriguez, M., Wessman, M.: Putting Teeth into Open Architectures: Infrastructure for Reducing the Need for Retesting. In: Proceedings of the 4th Annual Acquisition Research Symposium: Creating Synergy for Informed Change, Monterey, CA, May 16-17, pp. 285-312 (2007)

15. Dailey, P.: High-Fidelity Profile-Based Automated Testing of Open Architecture TrackProcessing Software, NPS Ph.D. Dissertation (June 2010)

16. Luqi, Goguen, J., Berzins, V.: Formal Support for Software Evolution. In: Proceedings of Monterey Workshop 1994, Monterey, CA, September 7-9, pp. 10-21 (1994) 
17. Luqi, Berzins, V.: Software Architecture in Computer-Aided Prototyping. In: Proceedings of 1995 Monterey Workshop on Increasing the Practical Impact of Formal Methods in Computer Aided Software Development: Software Architecture, Monterey, CA, September 12-14, pp. 44-57 (1995)

18. Luqi: Formal Models and Prototyping. In: Proceedings 1997 ARO Workshop on Requirements Targeting Software and System Engineering - Towards a Scientific Basis, Munich, Germany, October 12-14, pp. 183-194 (1997)

19. Luqi: Engineering Automation for Computer Based Systems. In: Proceedings of the 1998 ARO/ONR/NSF/DARPA Monterey Workshop on Engineering Automation for Computer Based Systems, Monterey, CA, April 1998, pp. 3-8 (1998)

20. Luqi, Nogueria, J.C.: A Risk Assessment Model for Evolutionary Software Projects. In: Proceedings of the 2000 Monterey Workshop on Modeling Software Systems Structures in a Fastly Moving Scenario, Santa Margherita Ligure, Italy, June 13-16, pp. 208-215 (2000)

21. Murrah, M., Johnson, C., Luqi: Enhancements \& Extensions of Formal Models for Risk Assessment in Software Projects. In: Proceedings Monterey Workshop 2001, Engineering Automation for Software Intensive System Integration, NPS, Monterey, CA, June 18-22, pp. 120-127 (2001)

22. Luqi, Qiao, Y., Zhang, L.: Computational Model for High-confidence Embedded System Development. In: Monterey Workshop - Radical Innovations of Software and Systems Engineering in the Future, Venice, Italy, October 7-11, pp. 265-303 (2002)

23. Luqi, Zhang, L.: Documentation Driven Agile Development for Systems of Embedded Systems. In: Monterey Workshop Series: Workshop on Software Engineering for Embedded Systems: From Requirement to Implementation, Chicago, IL, September 24-26, pp. 13-25 (2003)

24. 11th Monterey Workshop: Software Engineering Tools: Compatibility and Integration, Vienna, Austria (2004)

25. Luqi, Berzins, V., Roof, W.: Nautical Predictive Routing Protocol (NPRP) for the Dynamic Ad-Hoc Nautical Network (DANN). In: Monterey Workshop 2005: Realization of Reliable Systems on Top of Unreliable Networked Platforms, Univ. of California, Irvine, USA, Laguna Beach, September 22-24, pp. 1-9 (2005)

26. Luqi, Ivanchenko, V., Rodriguez, M., Berzins, V.: Reliability and Flexibility Properties of Models for Design and Run-time Analysis. In: Monterey Workshop 2006 Composition of Embedded Systems: Scientific and Industrial Issues, Paris, October 16-18 (2006)

27. 15th Monterey Workshop: Foundations of Computer Software, Future Trends and Techniques for Development, Budapest, Hungary (2008)

28. Demir, Y.: JOGL: An OpenGL Based Graphics Domain for Ptolemy II. In: Cyberphysical Systems Education Workshop (CPSEW), Arlington, VA, August 12 (2010)

29. Jacoby, G., Chang, D.: Towards Command and Control Networking of Cooperative Autonomous Robotics for Military Applications (CARMA). In: CCECE/CCGEI Conference, Niagara Falls, Canada, May 5-7 (2008)

30. Porter, A., Memon, A., Yilmaz, C., Schmidt, D., Natarajan, B.: Skoll: A Process and Infrastructure for Distributed Continuous Quality Assurance. IEEE Transactions on Software Engineering 33(8), 510-525 (2007)

31. Luqi, Dailey, P.: Profile-Based Automated Testing Process for Open Architecture TrackProcessing Software. Technical Report \#NPS-CS-10-005 (March 2010)

32. Niebuhr, D., Rausch, A., Klein, C., Reichmann, J., Schmid, R.: Achieving Dependable Component Bindings in Dynamic Adaptive Systems - A Runtime Testing Approach. In: Proc. Third IEEE International Conference on Self-Adapting and Self-Organizing Systems, San Francisco, CA, September 14-18, pp. 186-197 (2009) 
33. Prasanth, R., Boskovic, J., Mehra, R.: Computational Methods for the Verification of Adaptive Control Systems. In: Proc. SPIE 5429, 264, Orlando, FL, April 12, pp. 264-272 (2004)

34. Schaefer, I., Poetzsch-Heffter, A.: Model-Based Verification of Adaptive Embedded Systems under Environment Constraints. ACM SIGBED Review - Special Issue on the 2nd International Workshop on Adaptive and Reconfigurable Embedded Systems, APRES 2009, 6(3) (October 2009) (no page numbers used)

35. Zhang, J., Goldsby, H., Chang, B.: Modular Verification of Dynamically Adaptive Systems. In: Proc. AOSD 2009, Charlottesville VA, March 2-6, pp. 161-172 (2009) 